\title{
Diradicals acting through diamagnetic phenylene vinylene bridges: Raman spectroscopy as a probe to characterize spin delocalization
}

\author{
Sandra Rodríguez González, ${ }^{1}$ Belén Nieto-Ortega, ${ }^{1}$ Rafael C. González Cano, ${ }^{1}$ \\ Vega Lloveras, ${ }^{2,3}$ Juan J. Novoa, ${ }^{4}$ Fernando Mota, ${ }^{4}$ José Vidal-Gancedo, ${ }^{2,3}$ \\ Concepció Rovira, ${ }^{2,3}$ Jaume Veciana, ${ }^{2,3, a)}$ Elena del Corro, ${ }^{5}$ Mercedes Taravillo, ${ }^{5}$ \\ Valentín G. Baonza, ${ }^{5}$ Juan T. López Navarrete, ${ }^{1, a)}$ and Juan Casado ${ }^{1, a)}$ \\ ${ }^{1}$ Department of Physical Chemistry, University of Málaga, Campus de Teatinos s/n, Málaga 29071, Spain \\ ${ }^{2}$ Department of Molecular Nanoscience and Organic Materials, Institut de Ciència de Materials de Barcelona \\ (CSIC), Campus Universitari de Bellaterra, E-08193 Cerdanyola, Barcelona, Spain \\ ${ }^{3}$ NANOMOL group, Networking Research Center on Bioengineering, Biomaterials and Nanomedicine \\ (CIBER-BBN), Campus Universitari de Bellaterra, E-08193 Cerdanyola, Barcelona, Spain \\ ${ }^{4}$ Dpt. de Química Física and IQTCUB, Fac. Química, Univ. de Barcelona, Av. Diagonal, 647, \\ 08028-Barcelona, Spain \\ ${ }^{5}$ MALTA-Consolider Team, Department of Physical Chemistry, University Complutense of Madrid, \\ Madrid 28040, Spain
}

(Received 29 January 2014; accepted 8 April 2014; published online 25 April 2014)

\begin{abstract}
We present a complete Raman spectroscopic study in two structurally well-defined diradical species of different lengths incorporating oligo $p$-phenylene vinylene bridges between two polychlorinated triphenylmethyl radical units, a disposition that allows sizeable conjugation between the two radicals through and with the bridge. The spectroscopic data are interpreted and supported by quantum chemical calculations. We focus the attention on the Raman frequency changes, interpretable in terms of: (i) bridge length (conjugation length); (ii) bridge conformational structure; and (iii) electronic coupling between the terminal radical units with the bridge and through the bridge, which could delineate through-bond spin polarization, or spin delocalization. These items are addressed by using the "oligomer approach" in conjunction with pressure and temperature dependent Raman spectroscopic data. In summary, we have attempted to translate the well-known strategy to study the electron (charge) structure of $\pi$-conjugated molecules by Raman spectroscopy to the case of electron (spin) interactions via the spin delocalization mechanism. () 2014 AIP Publishing LLC. [http://dx.doi.org/10.1063/1.4871895]
\end{abstract}

\section{INTRODUCTION}

Magnetic coupling has been a central issue in Molecular Magnetism, with a great deal of attention in past years being devoted to the study of the different factors influencing the nature and strength of the magnetic coupling between spins connected through molecular bridges. ${ }^{1-3}$ Organic polyradicals and their simplest cases, diradicals, are very promising species for such studies, since the effective exchange interaction between their spins is generally much larger within the molecule (intramolecular magnetic coupling) than among neighboring molecules. Magnetism in these organic systems is attained by connecting "stable and persistent" radical units (i.e., chemical groups with one unpaired electron) to adequate molecular bridges, so promoting a magnetic coupling between the radical units via through-bond super-exchange interaction. ${ }^{1-6}$ The most promising linkers are $\pi$-conjugated bridges whose ability to promote electronic $\pi$-conjugation favors strong magnetic couplings or inter-spin interaction transmission. At the intramolecular level, there are two relevant mechanisms that make super-exchange magnetic coupling possible: ${ }^{7-9}$ (i) spin delocalization, in which the bridge

\footnotetext{
a) Author to whom correspondence should be addressed. Electronic addresses: vecianaj@icmab.es; teodomiro@uma.es; and casado@uma.es
}

connecting the radical centres makes the two spin sites to interact due to the delocalized nature of the bridge orbitals (when this mechanism operates, the geometrical properties of the bonds connecting the radicals with the bridge and within the bridge can significantly change regarding the unsubstituted bridge with a measurable impact on the molecular vibrational properties). (ii) spin polarization, controlled by topological or alternation rules, in which the number of carbon atoms between the radical centres determines the sign of exchange interaction for a odd number of atoms the ground state is high spin, if even, low spin.

The inclusion of a conjugated linker mediating the magnetic properties of a molecule may have additional benefits related with: (i) the semiconducting properties of the conjugated wire itself (conjugated wires have been successfully incorporated in organic field effect transistors ${ }^{10-12}$ ); (ii) the possibility of combining spin and electron transport through molecular wires, which is an important issue for spintronic applications, ${ }^{13,14}$ and (iii) the possibility to combine luminescent properties together with magnetism if photoactive bridges, such as $p$-phenylene vinylene, are used as linkers of the radical centres. ${ }^{15-20}$

Some of us have reported series of structurally welldefined diradicals ${ }^{21,22}$ some of them incorporating oligo-pphenylene vinylenes (PPV) as bridges with different lengths, 

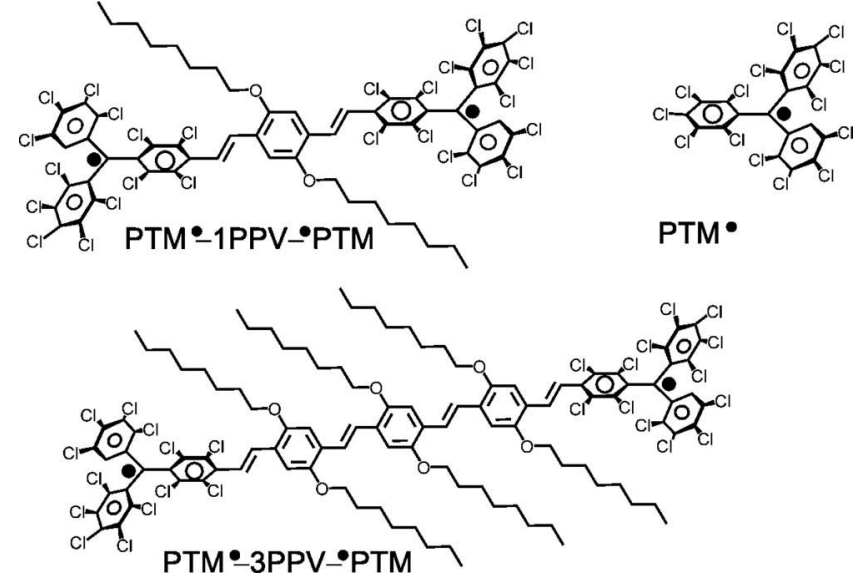

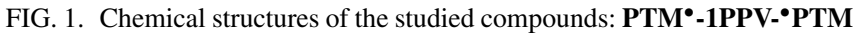
and PTM $^{\bullet}$-3PPV- $\bullet$ PTM diradicals and $\mathbf{P T M}^{\bullet}$ radical.

substituted with two solubilising long alkoxyl groups at the ortho positions of all 1,4-phenylene rings and terminally substituted with polychorotriphenylmethyl (PTM) radical units. $^{23,24}$ Such diradicals $\left(\mathbf{P T M}^{\bullet}-\mathbf{n P P V}-{ }^{\bullet} \mathbf{P T M}\right.$, see Figure 1) were partially reduced using chemical or electrochemical means, yielding the corresponding radical-anions. Such mixed-valence species enabled to study the long-range intramolecular electron transfer (IET) in their ground states, via tunnelling and hopping mechanisms, and the influence of the oligo-PPV bridge lengths and of the temperature and solvent nature on the IET phenomenon. ${ }^{23,24}$ However, the magnetic couplings and spin delocalization in this family of diradicals remain unknown. Our challenge here is to understand the role played by the diamagnetic conjugated PPV bridge in the magnetic coupling in some of such diradicals. In particular, we shall focus on the impact of the spin delocalization mechanism in two diradicals members of this series, those with one and three PPV units (depicted in Figure 1). Although these diradical entities are constructed with the same spin polarization concept, they show different inter-radical distances and different inter-radical $\pi$-electron delocalizations, either promoted by a different bridge conformation or by a different number of $\pi$-electrons in the diamagnetic union. Nonetheless, these molecules provide a perfect case for the straightforward study of the spin delocalization effect in diradicals and to get further insight into the effects that could control it (i.e., bridge conformation, radical-to-bridge conjugation, and number of $\pi$-conjugated electrons). Given the central role of conjugation (i.e., $\pi$-electron delocalization), we take advantage of using Raman spectroscopy as a probe. Several reasons justify the choice of this experimental technique: (i) Raman spectroscopy is well known and broadly used for the characterization of the electronic (electron charge) structure of $\pi$-conjugated molecules, ${ }^{25,26}$ (ii) comparison of the vibrational Raman spectra of different compounds will directly account for the effect of radical-to-bridge conjugation in the radical and bridge structures which, if promoted by the unpaired electrons, can contain the effect of spin delocalization. (iii) Raman spectroscopy is greatly suited to measure the vibrational spectra in the solid state and can be successfully coupled to complementary techniques like high pressure cells and variable temperature stages. High pressure studies provide a clean physical way to investigate the influence of backbone conformation, since pressure is able to tune the geometry of the flexible molecular wires without any modification of the composition. ${ }^{27-31}$ Raman spectroscopy can also be easily combined with variable temperature experiments, which have revealed very important to elucidate the magnetic behaviour; and (iv) we recently demonstrated the power of Raman spectroscopy for the study of diradical compounds acting through conjugated thienyl and phenylene bridges. $^{32,33}$ Finally, we shall use quantum chemical calculations to support and interpret the vibrational spectroscopy results.

\section{EXPERIMENTAL AND THEORETICAL DETAILS}

\section{A. Experimental measurements}

FT-Raman spectra: $1064 \mathrm{~nm}$ FT-Raman spectra were measured using a FT-Raman accessory kit (FRA/106-S) of a Bruker Equinox 55 FT-IR interferometer. A continuouswave Nd-YAG laser working at $1064 \mathrm{~nm}$ was employed for excitation. Resonance Raman spectra: 785, 633, and $532 \mathrm{~nm}$ Resonance Raman spectra were recorded by using a Senterra dispersive Raman spectrometer from Bruker. High-pressure Raman spectroscopy: A modified Merrill-Basset sapphire anvil cell was coupled to a ISA HR460 spectrograph using a 100x Mitutoyo objective. ${ }^{34}$ Raman spectra were excited with a Spectra-Physics solid-state laser operating at $532 \mathrm{~nm}$. High pressure measurements were performed under both hydrostatic and non-hydrostatic conditions in order to analyze whether the molecular geometry is affected or not by residual stresses. Non-hydrostatic experiments were carried out by compressing the sample deposited onto a copper gasket located between the two anvils. Hydrostatic measurements were performed using a 4:1 methanol-ethanol mixture as pressure medium in a sample chamber practiced on the copper gasket. Pressures were measured using the ruby luminescence technique ${ }^{35}$ with an accuracy of 1 kbar. Variable-temperature Raman spectra: A variabletemperature cell Specac P/N 21525, with interchangeable pairs of quartz windows, was used to record the FT-Raman spectra at different temperatures. A Linkam cell equipped for the Senterra Raman spectrometer microscope was used for the measurements at $633 \mathrm{~nm}$ excitation wavelength. Electron Spin Resonance spectra: ESR spectra were obtained in an X-band spectrometer (Bruker ESP $300 \mathrm{E}$ ) equipped with a field-frequency $(\mathrm{F} / \mathrm{F})$ lock accessory and built in NMR Gaussmeter. A rectangular TE102 cavity was used for the measurements. The signal to noise ratio of spectra was increased by accumulation of scans using the F/F lock accessory to guarantee large field reproducibility. Precautions to avoid undesirable spectral distortions and line broadenings, such as those arising from microwave power saturation and magnetic field over modulation, were also taken into account. To avoid dipolar line broadening from dissolved oxygen, solutions were always carefully degassed with pure Argon. 


\section{B. Theoretical calculations}

Calculations were done in the framework of the Density Functional Theory (DFT) ${ }^{36}$ with the Gaussian03 package of programs. ${ }^{37}$ The Becke's three parameter (B3) gradientcorrected exchange functional combined with the correlation Lee-Yang-Parr (LYP) functional was used. ${ }^{38,39}$ We have also carried out calculations with the new LCBOP functional to put in context the use of the B3LYP results. LCBOP stands for "Long range Carbon Bond Order Potential" and represents a bond order potential that includes long-range dispersive and repulsive interactions. ${ }^{40}$ The $6-31 \mathrm{G}^{* *}$ basis set was used. ${ }^{41}$ Octyloxy side groups were fully removed in the calculations which leaves unaffected the number of electrons in the $\pi$-space. No other constraints have been imposed during the geometries optimization. For the open shell singlet biradicals, the broken symmetry approach (guess $=$ mix keyword) was considered in all the cases, while for the triplets the UHF approach was applied to avoid spin contamination. ${ }^{42}$ Each spin state was independently optimized. Theoretical Raman spectra were obtained for the resulting ground-state optimized geometries, harmonic vibrational frequencies, and Raman intensities were calculated numerically at the same level of calculation and scaled by a factor of $0.96 .{ }^{43}$ Theoretical Raman spectra were simulated by using gaussians functions (FWHH, $\left.\Delta 3 \mathrm{~cm}^{-1}\right)$.

\section{Synthesis}

The synthesis of the samples studied here have been reported in Refs. 23 and 24.

\section{RESULTS AND DISCUSSION}

\section{A. Theoretical structures and singlet-triplet analysis}

A straightforward support of the coupling between the two unpaired spins in the two PTM radical centers can be given by calculating the singlet-triplet splitting, $\Delta\left(\mathrm{S}_{0^{-}}\right.$ $\mathrm{T}_{1}$ ). Three possible electronic configurations were evaluated at the DFT/6-31G** level, either with the long-range (U)LC-BOP or with the standard (U)B3LYP functionals (see Figure 2): the closed-shell case (i.e., the two spins are paired in the same orbital), the singlet diradical (i.e., the two spins are paired but in different disjoint orbitals, using the broken symmetry option), and the triplet diradical (i.e., unpaired spins). From these calculations the $\Delta\left(\mathrm{S}_{0}-\mathrm{T}_{1}\right)$ can be derived, Figure 2. $\Delta\left(\mathrm{S}_{0}-\mathrm{T}_{1}\right)$ corresponding to strong (covalently bonded electrons) in $\pi$-conjugated molecules is rather large. In the opposite case, for those radicals interacting weakly, one expects this splitting to be small but non-negligible. From these theoretical $\Delta\left(\mathrm{S}_{0}-\mathrm{T}_{1}\right)$ values, the following can be deduced: (i) the closed-shell configuration is rather unstable due to the energy cost for the rupture of the aromaticity of the benzene units in the bridge; (ii) in all the cases the open-shell singlet diradical structure is the most stable $\left(\mathrm{S}_{0}\right)$ electronic configuration with the first triplet excited state ( $\left.\mathrm{T}_{1}\right)$ always placed at slightly higher energies; (iii) $\Delta\left(\mathrm{S}_{0}-\mathrm{T}_{1}\right)$ for PTM-1PPV-'PTM are small, 0.036 and $0.031 \mathrm{kcal} / \mathrm{mol}$ at the (U)B3LYP/6-31G** and (U)LC-BOP/6-31G** levels, respectively (see Figure 2).

As it was expected, the $\Delta\left(\mathrm{S}_{0}-\mathrm{T}_{1}\right)$ values decrease with the enlargement of the bridge. Also in Figure 2, the topologies of the SOMOs of PTM $\mathbf{P}^{\bullet} \mathbf{- 1 P P V}-\boldsymbol{\bullet P T M}^{-P}$ show that some electron densities are placed on the bridge between the two external spin bearing PTM units which is indicative of certain inter-spin interaction.

\section{B. Experimental and theoretical Raman spectra}

Let us first comment the evolution of the molecular structures based on the optimized geometries for PTM・-1PPV-

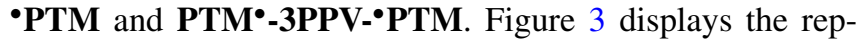
resentative structures of the open singlet and triplet species of PTM-1PPV-・PTM. These indicate that the dihedral angles between the PTM moieties and the closest ethylenic bridge are large (i.e., $\approx 47^{\circ}$ ) and keep constant with the enlargement of the bridge. In contrast, the dihedral angles between the phenyls and the ethylenes in the spacer are small $\left(\approx 7^{\circ}-8^{\circ}\right)$ and become slightly smaller (more planar) by increasing the spacer length. For $\mathbf{P T M}^{\bullet} \mathbf{- 1 P P V -} \mathbf{P}^{\bullet} \mathbf{P T M}$ the differences between the singlet and triplet open shells structures are small and predict an enlargement of the planarity distortion for the triplet species.

For a given level of calculus, the theoretical Raman spectra of PTM $^{\bullet}$-1PPV- ${ }^{-P T M}$ in its singlet open shell biradical and triplet states were calculated using the lower energy electronic configurations at the DFT/(U)LC-BOP/6-31G** and DFT/(U)B3LYP/6-31G** levels which are shown in Figure 3
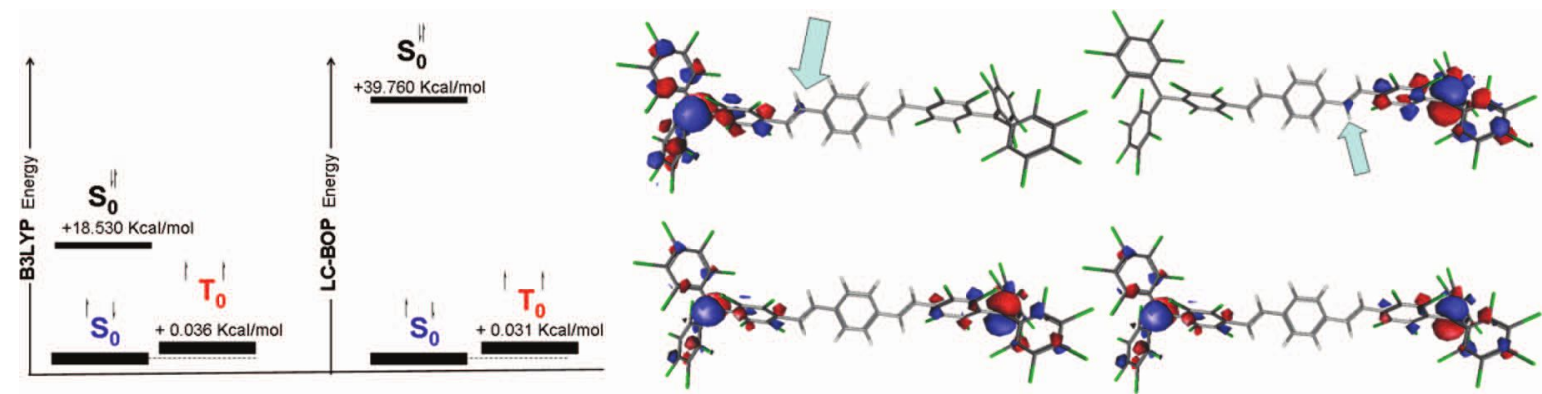

FIG. 2. (Left) Ground and low-lying excited electronic state energies predicted theoretically at the DFT/(U)B3LYP (left) and DFT/(U)LC-BOP/6-31G** (right) levels for $\mathbf{T P M}^{\bullet}$-1PPV-・PTM. (Right) Orbital topologies of the magnetic SOMO-1 (left) and SOMO-2 (right) of the open-shell singlet diradical (top) and triplet diradical (bottom) configurations of PTM $\mathbf{P}^{\bullet}$-1PPV-・PTM. The arrows highlight the contribution of the bridge. Isosurface values of the plots, \pm 0.02 . 

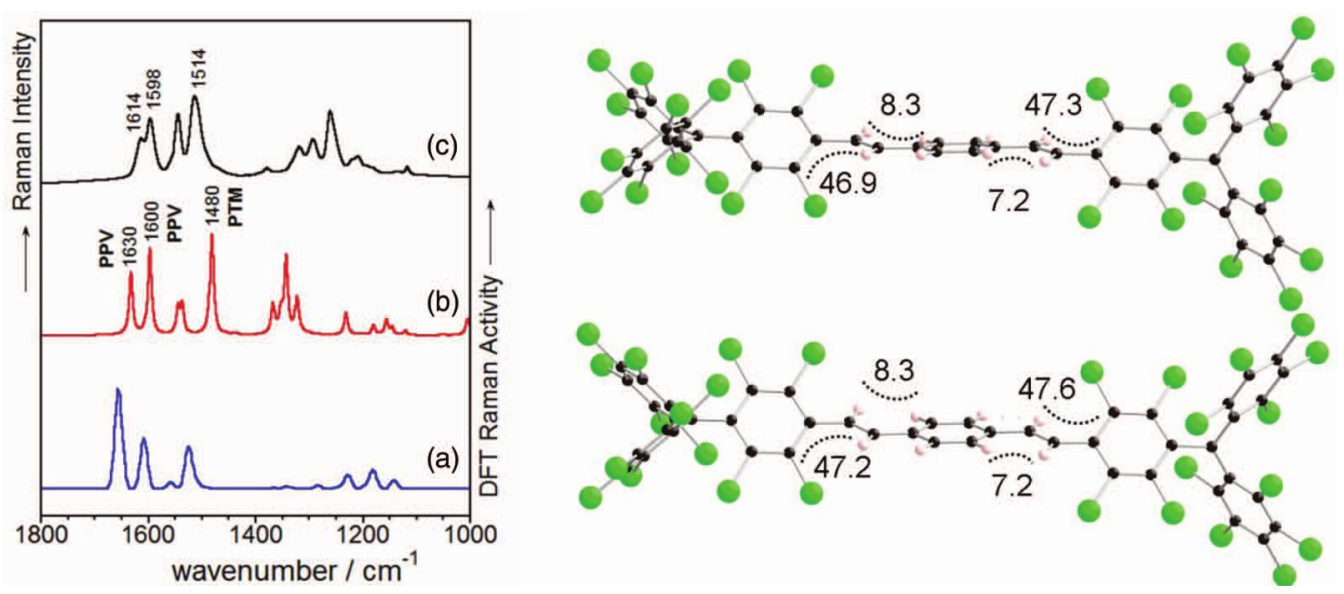

FIG. 3. (Left) Raman spectra for $\mathbf{P T M}^{\bullet}-\mathbf{- 1 P P V}-\bullet^{-P T M}$ : (a) calculated at the DFT/(U)LC-BOP/6-31G** level for the triplet diradical state, (b) calculated at the DFT/(U)B3LYP/6-31G** level for the triplet diradical state, and (c) experimental FT-Raman spectrum in solid state at room temperature. (Right) Energy optimized structures for PTM $^{\bullet}$-1PPV-・PTM in its singlet (top) and triplet (bottom) open shell configurations at the DFT/(U)LC-BOP/6-31G** level (numerical values correspond to the dihedral angles). See Figure $\mathrm{S} 2$ of the supplementary material ${ }^{49}$ for the vibrational eigenvectors.

together with the experimental FT-Raman spectrum. Much more resemblance between the experimental spectrum and that at the DFT/(U)B3LYP/6-31G** level is observed. In addition, the theoretical spectra are practically identical for the open shell biradical and triplet considering the same level of calculus (Figure S1 of the supplementary material), ${ }^{49}$ hence, in the following we will present only the theoretical spectra in the triplet state. The main features of the experimental spectrum are well reproduced by theoretical calculations. The two theoretical Raman modes around $1630 / 1600 \mathrm{~cm}^{-1}$, calculated for $\mathbf{P T M}^{\bullet} \mathbf{- 1 P P V}-\mathbf{P T M}^{-}$, correlate well with the pair of experimental bands observed at $1614 / 1598 \mathrm{~cm}^{-1}$ which are due to CC stretching modes of the central $p$ phenylene-vinylene bridge (see their vibrational eigenvectors in Figure S2 of the supplementary material). ${ }^{49}$ In particular, the theoretical one at $1630 \mathrm{~cm}^{-1}$ mostly arises from stretches of the vinylene group, while in the band around $1600 \mathrm{~cm}^{-1}$ the central benzene is more involved in the vibrational mode. The calculated band close to $1480 \mathrm{~cm}^{-1}$ has its experimental counterpart at $1514 \mathrm{~cm}^{-1}$ and arises from a stretching mode of the lateral polychlorinated benzene groups, directly connected to the central PPV bridge. According to calculations, the band centered at $1550 \mathrm{~cm}^{-1}$, experimentally found at $1544 \mathrm{~cm}^{-1}$, is predicted to be a mixed $v(\mathrm{CC})+\beta(\mathrm{CH})$ vibrational mode delocalized from the external PTM radical towards the central bridge. We will recognize this band to be a mostly PTM radical based vibrational mode in Sec. III C by taking into account the oligomer approach evolution.

The recognition of Raman modes on the external PTM radical and in the central bridge provides a means to analyze the electronic effects taking place in the molecule as a consequence of the existence of inter-PTM spin coupling and its modulation with the length of the spacer. In the following, we will attempt to correlate the changes in the Raman spectra of the diradical species with the interaction between the spin bearing units at the termini with the hope of getting insights on the through-bond bridge spin delocalization, which must be more effective in a spin format that enhances the role of the bridge.

\section{FT-Raman and EPR spectra and the "oligomer approach"}

The effect of the distance on the inter-radical coupling is critical on the basis of the arguments of any magnetic mechanism. Consequently, it is worth to study such a dependence using a series of analogous diradicals differing in their interradical separation; i.e., the so-called "oligomer approach." 4 Figure 4 displays the experimental FT-Raman spectra of PTM $^{\bullet}$-1PPV-・PTM, PTM ${ }^{\bullet}-3 P P V-{ }^{\bullet} \mathbf{P T M}$ and PTM $^{\bullet}$. The elongation of the oligo-PPV fragment leads to an enhancement of the Raman lines associated with the central bridge (1614 and $1598 \mathrm{~cm}^{-1}$ ), however their frequencies upshift and downshift to 1621 and $1587 \mathrm{~cm}^{-1}$, respectively. In contrast, the band related with the external spin bearing PTM moieties appear at $1509 \mathrm{~cm}^{-1}$ in PTM', $1514 \mathrm{~cm}^{-1}$ in PTM'-1PPV-PTM, and $1518 \mathrm{~cm}^{-1}$ in PTM $^{\bullet}-\mathbf{3 P P V}-\boldsymbol{P P T M}^{25,33,34}$

A possible interpretation of this finding is based on the well-known electron acceptor character of the external

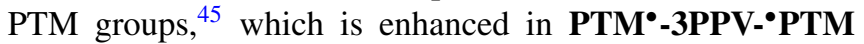

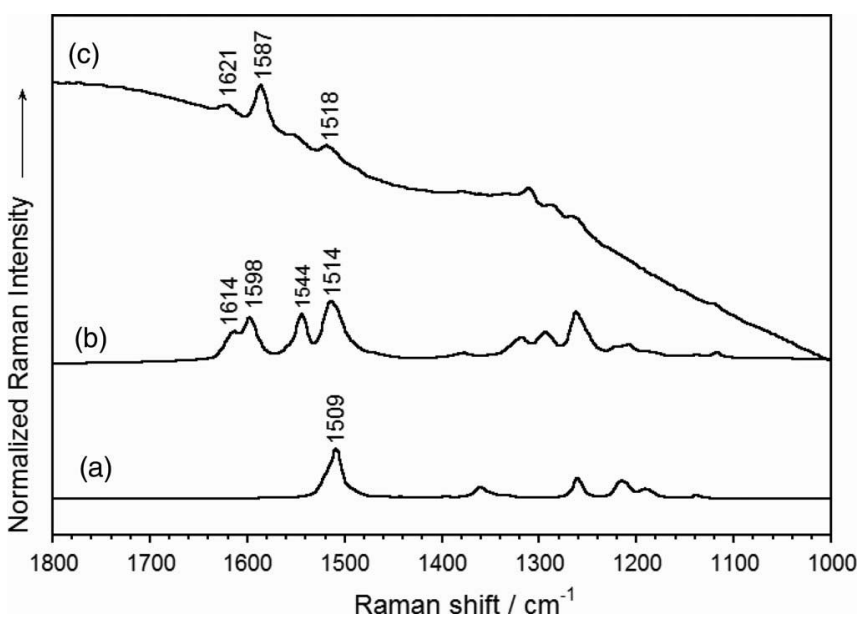

FIG. 4. FT-Raman spectra of (a) $\mathbf{P T M}^{\bullet}$, (b) $\mathbf{P T M}^{\bullet}-\mathbf{1 P V}-\boldsymbol{\bullet}^{\mathbf{P T M}}$, and of (c) PTM $^{\bullet}-3 P V-{ }^{\bullet} \mathbf{P T M}$ in the solid state. 
regarding PTM $^{\bullet}$-1PPV- ${ }^{\bullet}$ PTM since the PTM radical exerts a more profound electron withdrawal over a richer (i.e., larger) electron donor bridge, hence provoking a Raman upshift of their vibrational frequencies. Simultaneously, this electron release towards the end acceptor groups makes the PPV moiety to get more quinoidized producing the Raman downshift. This Raman frequency behavior clearly denotes the existence of an effective $\pi$-electron delocalization over the whole central bridge and the terminal spin bearing centers by an Acceptor-Donor-Acceptor charge-polarization interaction. The evolution of the spectra with the bridge length allows us to clarify the origin of the experimental band at $1544 \mathrm{~cm}^{-1}$ which clearly decreases its relative intensity regarding the pure bridge vibrations around $1600 \mathrm{~cm}^{-1}$ on

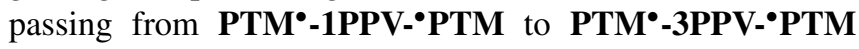
indicative of the main location of this mode on the PTM units.

In line with the presence of an effective $\pi$-electron delocalization over the whole molecule is the strength of the dipolar magnetic interaction between the two unpaired electrons

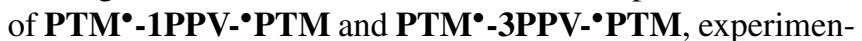
tally observed. Figure 5 shows the experimental X-band EPR spectra of both diradicals in a frozen $\mathrm{CH}_{2} \mathrm{Cl}_{2}$ :toluene (1:1) mixture at $140 \mathrm{~K}$, as well as their simulations using the anisotropic components of $g$ factor and the zero-field splitting parameters $/ D^{\prime} /$ and $/ E^{\prime} /$, given in Table I. Parameter $/ D^{\prime} /$ can be used to estimate an effective inter-spin distance $(R$, in $\AA$ ), assuming that the point magnetic dipole approach, where $/ D^{\prime} /=3 g^{2} \beta^{2} / 2 R^{3},{ }^{46}$ is valid for these conjugated diradicals. In both cases the estimated effective distances are smaller than the nominal distances between the central C atoms of PTM units, obtained from DFT calculations, revealing therefore the extension of the delocalization of the two unpaired electrons on the molecular skeleton.
TABLE I. EPR parameters used for the simulation of experimental X-band spectra of studied diradicals. $^{\text {a }}$

\begin{tabular}{lccccccc}
\hline \hline Diradical & $g_{x}$ & $g_{y}$ & $g_{z}$ & $\begin{array}{r}\text { Gauss) } \\
/ D^{\prime} /(\text { in }\end{array}$ & $\begin{array}{l}/ E^{\prime} /(\text { in } \\
\text { Gauss })\end{array}$ & $R($ in $\AA)$ & $\begin{array}{c}R_{\text {nom }} \\
\text { (in } \AA)\end{array}$ \\
\hline $1 \mathrm{PPV}$ & 2.0027 & 2.0025 & 2.0018 & 9.0 & $\sim 0$ & 14.6 & 19.2 \\
$3 \mathrm{PPV}^{\mathrm{b}}$ & 2.0040 & 2.0018 & 2.0012 & $\sim 1.2$ & $\sim 0$ & $\sim 28.5$ & 32.5 \\
\hline \hline
\end{tabular}

${ }^{\mathrm{a}}$ For both simulations hyperfine couplings components of $\mathrm{A}_{\mathrm{xx}}\left(=\mathrm{A}_{\mathrm{yy}}=\mathrm{A}_{\mathrm{zz}}\right)$ of 0.88 Gauss with 2 equivalent $\mathrm{H}$ nuclei were used.

${ }^{\mathrm{b}}$ The lack of a more defined spectral features in this spectrum yields parameters with a poor precision for this diradical. The use of EPR spectra with higher frequency bands would be required to get more precise values.

\section{Resonance Raman spectroscopy}

Related with the last discussion, the UV-Vis absorption spectra of the two symmetric compounds display an intense band at $388 \mathrm{~nm}$ characteristic of PTM radical chromophores along with other electronic absorptions appearing at 509/680 $\mathrm{nm}$ in PTM $^{\bullet}$-1PPV- ${ }^{-}$PTM (see Figure 6) and at

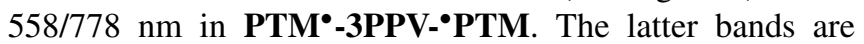
assigned to the intramolecular charge-transfer from the central PPV electron-donor bridge towards the external PTM electron-acceptor units promoted by the perchlorination of these units.

Resonance Raman spectra obtained when the excitation wavelength coincides with an electronic absorption informs us about the molecular segments mostly involved in the band excited in the Raman experiment. The comparison of resonant (532 and $633 \mathrm{~nm}$ ) and non-resonant $(1064 \mathrm{~nm}) \mathrm{Ra}-$ man spectra in Figure 6 for PTM $^{\bullet}$-1PPV-・PTM reveals an enhancement by resonance of the $p$-phenylene vibration at $1598 \mathrm{~cm}^{-1}$. Compared to the PTM radical vibrations, the band at $1614 \mathrm{~cm}^{-1}$, mostly due to the vinylene bridge connections,
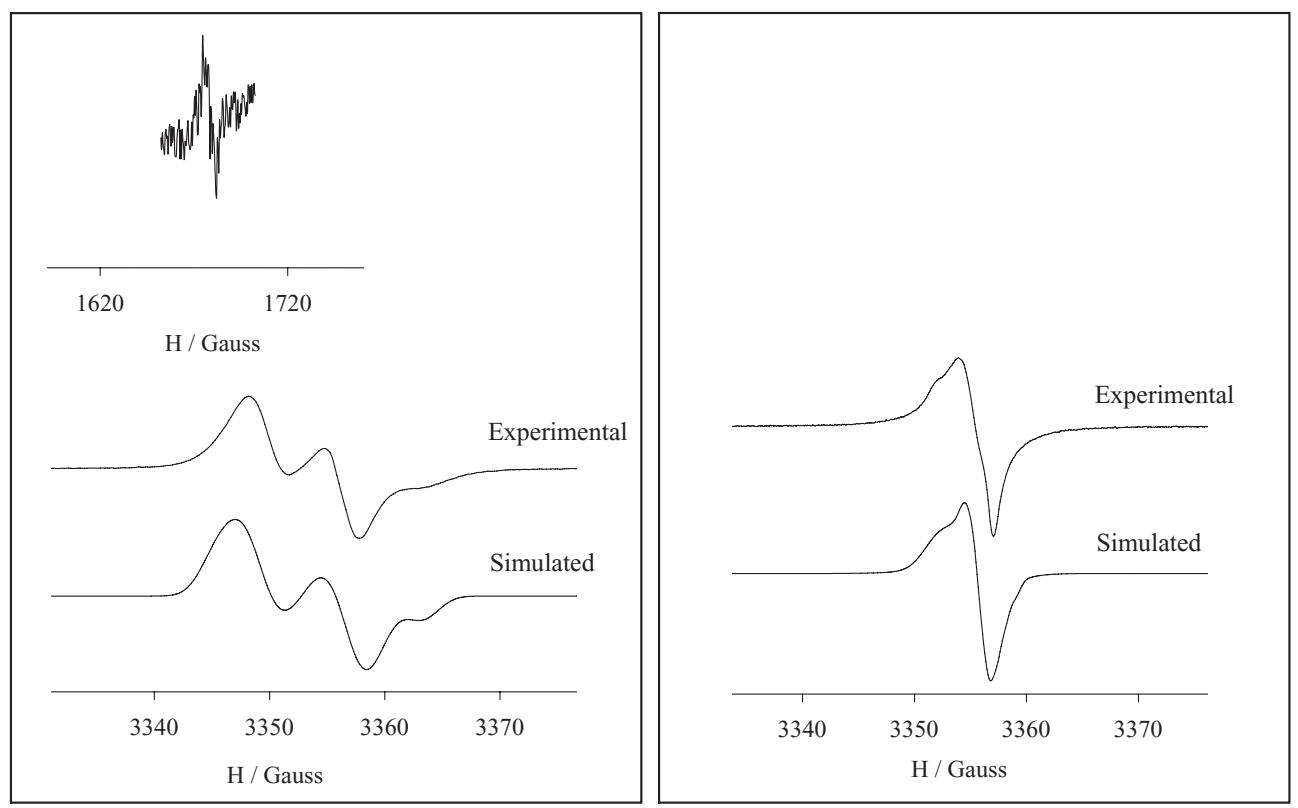

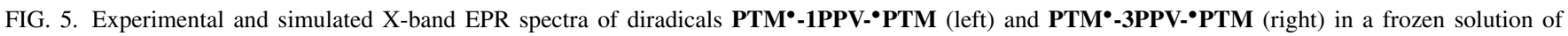
toluene:dichloromethane (1:1) at $140 \mathrm{~K}$. (Inset) Half-field band of the diradical $\mathbf{P T M}^{\bullet} \mathbf{\bullet} \mathbf{1 P P V}-\boldsymbol{\bullet}^{\mathbf{P T M}}$ under the same conditions. Simulations were performed using the data given in Table I. 

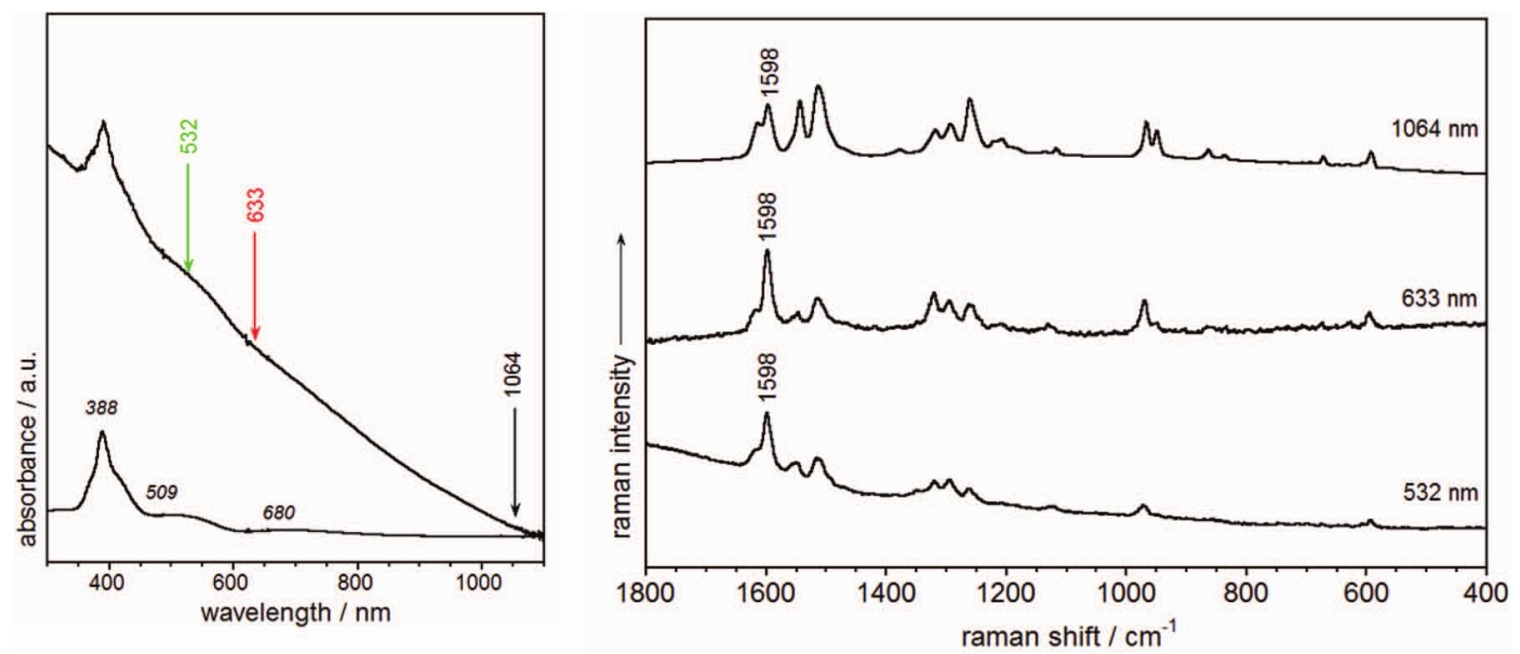

FIG. 6. (Left) UV-Vis absorption spectra in $\mathrm{CH}_{2} \mathrm{Cl}_{2}$ solution (bottom) and in the solid state (top) of PTM・-1PPV-・PTM. (Right) Solid state Raman spectra of PTM*1PPV-・PTM at different excitation wavelengths.

is also enhanced by resonance, though to a less extent compared to the phenylene mode.

In all the cases, the study of the dependence of the Raman spectra with the excitation wavelength indicates that both the PPV bridge and the PTM spin bearing moieties are intensively involved in the relevant $\pi-\pi *$ electron excitation and in the magnetic orbitals, hence electronic and spin excitations surely involve the electronic structure of the "diamagnetic" bridge. ${ }^{24}$

Given the bridge to external electron-deficient sites charge-transfer character of the low lying electronic absorptions, and the selective Raman enhancement of the $1598 \mathrm{~cm}^{-1}$ mode located in the 2,5-dialkyloxy $p$-phenylene bridge, it can be stated that this bridge controls or modulates the efficiency of electron communication between the two external PTM groups. The phenyl rings can be viewed as the bottleneck point for inter-radical conjugation since, on one hand, the aromatic character of benzene prevents an extensive $\pi$-electron delocalization and, on the other hand, the possible distortions relative to the plane perpendicular to the two $p_{z}$ atomic orbitals with the unpaired electrons could affect the conjugation (see Figure 3).$^{47}$ These two features are critical for a convenient spin delocalization and magnetic coupling between the two spins in these diradicals. We can therefore use the band appearing at $1598-1587 \mathrm{~cm}^{-1}$ to analyze the effect on the conjugational properties of the bridge conformation and bridge lengthening. Importantly, this Raman band is incisively affected by external pressure, as it is discussed below.

\section{E. Pressure dependence of Raman spectra}

It is well documented that in oligophenyl oligomers there are two bands around $1220-1240 \mathrm{~cm}^{-1}$ and $1280-1320 \mathrm{~cm}^{-1}$ owing to $\beta(\mathrm{CH})$ and $v(\mathrm{C}-\mathrm{C})$ modes whose relative Raman intensity decreases as the oligomer backbone becomes planar. This effect can be observed in high-pressure studies, ${ }^{10}$ where repulsive interactions force the molecules to acquire an almost planar geometry at moderate pressures. To the best of our knowledge, there is one similar study reported in the literature dealing with oligoPPVs. ${ }^{48}$
Figure 7 compares the $532 \mathrm{~nm}$ Raman spectra of PTM3PPV-・PTM at high pressure, under both hydrostatic and non-hydrostatic conditions, and the evolution of the spectra at increasing non-hydrostatic pressures. Spectra were corrected for a small luminescence background and subsequently scaled for comparison. Trying to find out a similar behavior to that described in their parent oligophenyls, we analyzed the relative intensity of the bands located around 1286 and $1311 \mathrm{~cm}^{-1}$ that, according to our theoretical calculations, can be described by mixed $\beta(\mathrm{CH})+v(\mathrm{C}-\mathrm{C})$ movements. Results, in Figure 7 , show that the Raman intensity ratio $\left(\mathrm{I}_{1311} / \mathrm{I}_{1286}\right)$ slightly decreases with increasing pressure, which can be related to the planarization of the backbone. This is in nice agreement with theoretical calculations, which predict the conformation of the central bridge to be distorted from the co-planarity in the gas-isolated phases considered in the model. In addition, the band around $1550 \mathrm{~cm}^{-1}$ is largely intensified by pressure in comparison to the $1580-1600 \mathrm{~cm}^{-1}$ bands (Figure 7 right). This band is barely observable in the $532 \mathrm{~nm}$ Raman spectrum at ambient conditions and becomes a
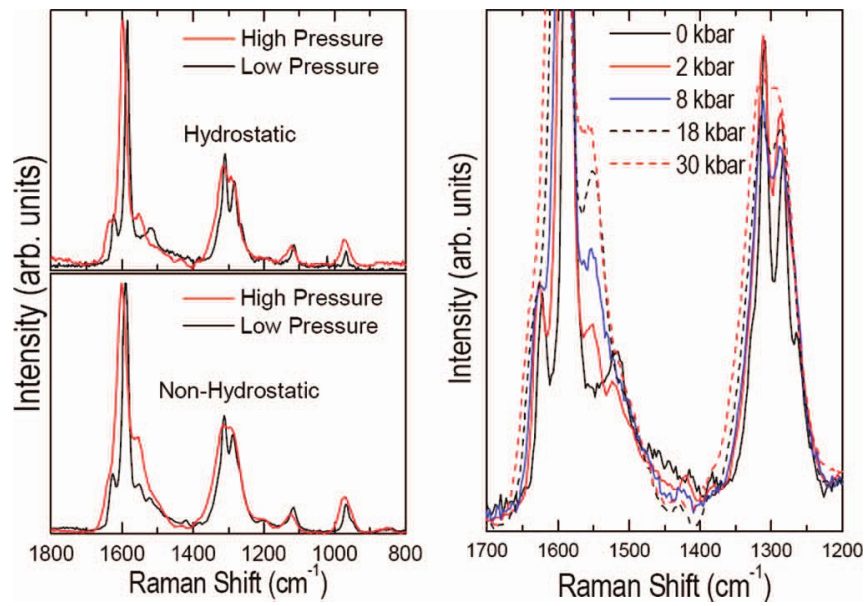

FIG. 7. Raman spectra of $\mathbf{P T M}^{\bullet}-\mathbf{3 P P V}-\mathbf{P T M}^{-}$in the solid state at selected pressures under both hydrostatic and non-hydrostatic conditions (see text). Spectra were excited at $532 \mathrm{~nm}$ and are normalized to the $1600 \mathrm{~cm}^{-1}$ band. 

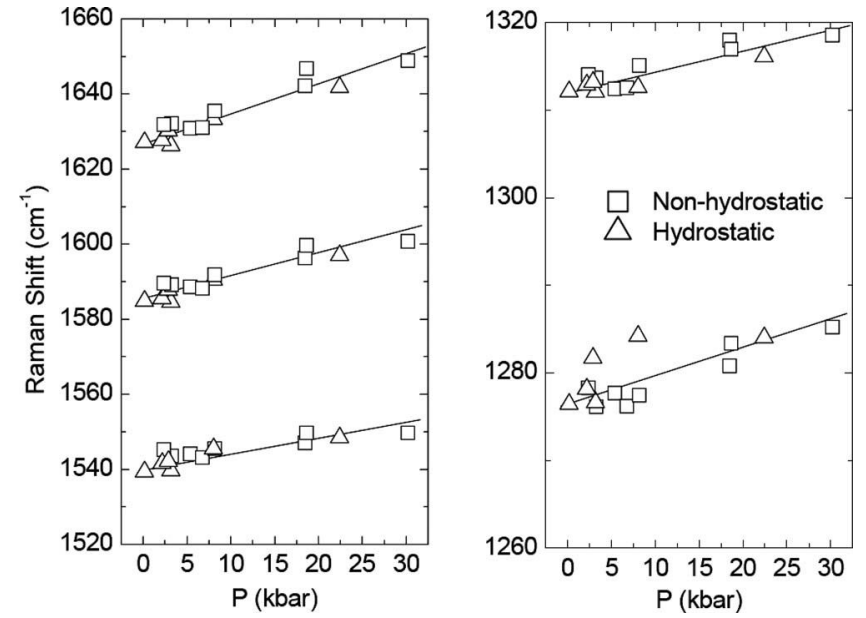

FIG. 8. Raman frequencies of the most intense features of $\mathbf{P T M}^{\bullet}-\mathbf{3 P P V}$ -PTM in the solid state under both hydrostatic and non-hydrostatic conditions (see text). Spectra were excited at $532 \mathrm{~nm}$.

medium intensity feature under compression. Due to its PTM radical character and its $\mathrm{CC}$ stretching nature its enhancement with pressure is likely due to the favored $\pi$-electron delocalization towards the PPV bridge in a more planar segment. In the hydrostatic regime, the PTM radical bands at 1550 and $1520 \mathrm{~cm}^{-1}$ of the PTM groups further increase their intensity, what, such as already stated, might be another indication of the favored participation in the $\pi$-electron and spin delocalization of these PTM radical groups with increasing pressure (due to greater planarization) anticipating a favourable situation for larger of magnetic coupling through the diamagnetic bridge.

Aside of the changes in the relative intensities of the Raman bands, the most apparent effects of pressure on the Raman spectra of molecular systems are band broadening (e.g., the band around $1620 \mathrm{~cm}^{-1}$ ) and upshifts of the spectral features with increasing pressure. This latter effect is analyzed in Figure 8, where the frequencies of the most intense bands of PTM $^{\bullet}$-3PPV- ${ }^{\bullet}$ PTM are plotted as a function of pressure. The pressure shifts are in good agreement with existing results on similar polymeric compounds. ${ }^{10}$ We observe that the most intense spectral feature appearing at $1585 \mathrm{~cm}^{-1}$ at room pressure shifts to $1600 \mathrm{~cm}^{-1}$ around $30 \mathrm{kbar}$, close to that observed in $\mathbf{P T M}^{\bullet}-\mathbf{1 P P V}-\mathbf{P P T M}^{-}\left(1598 \mathrm{~cm}^{-1}\right)$. This is again in consonance with a more pronounced planarization effect in the $\mathbf{P T M}^{\bullet}-\mathbf{3 P P V}-\mathbf{P}^{-} \mathbf{P T M}$ bridge which becomes similar to that of the shorter homologue $\mathbf{P T M}^{\bullet}$-1PPV- ${ }^{\bullet} \mathbf{P T M}$ (see theoretical calculations in Sec. III B).

It is interesting to address the evolution of the Raman spectra with increasing pressure with respect to the shape of the ground state potential energy surface depicted in Figure 9, where two possible shapes for the potential energy surface of the ground electronic state ( $W$ vs. $U$ ) are outlined: (i) in the case of the $U$ shape, the conformational energy $(\Delta \mathrm{E})$ is overcome by the magnetic interaction, yielding a total $\Delta \mathrm{E} \approx 0$ (energy barrier between the two local minima) with a full ground electronic delocalized system; (ii) in the $W$ shape case, the exchange energy is small and the system adopts two minima or localized spins in the PTM units. These

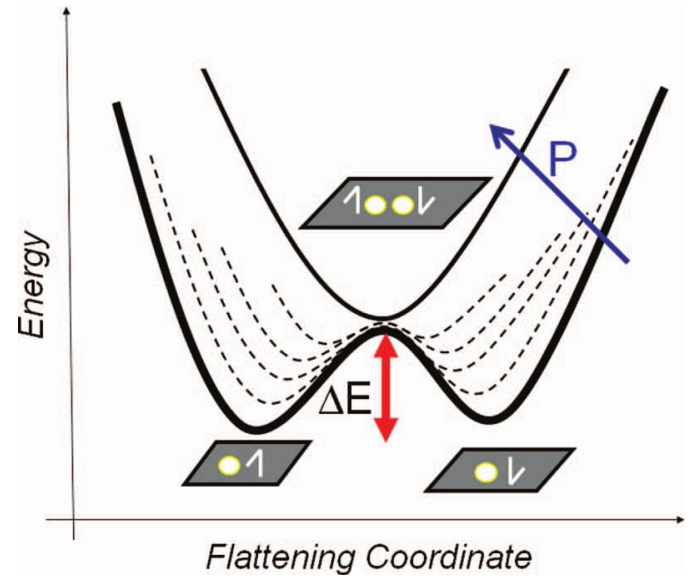

FIG. 9. Sketch of potential energy surfaces controlled by the spin coupling and the evolution with the flattening of the bridge structure between the two subunits.

two $W$ vs. $U$ situations are extreme cases and our systems are placed in between. Given that pressure induces the flattening of the structure, it would reduce $\Delta \mathrm{E}$ meaning a transformation from the $W$-like shape to $U$-like shape. Thus, the increment of $\pi$-electron delocalization upon pressure flattening, the similitude between the spectra of the short and long bridge molecules (meaning inter-radical distance reduction due to through-bond spin delocalization), and the larger involvement of the PTM radical groups in the whole Raman signal at high pressure might be indications of the ground electronic state $W \rightarrow U$ shift.

\section{F. Variable temperature Raman spectra}

The Raman spectra of $\mathbf{P T M}^{\bullet}-\mathbf{1 P P V}-\boldsymbol{P}^{\mathbf{P T M}}$ as a function of the temperature are shown in Figure 10. Though no dramatic changes are observed between $-170^{\circ} \mathrm{C}$ and $200^{\circ} \mathrm{C}$, two main features are observed between the lowest and highest temperature spectra: (i) the feature at $1598 \mathrm{~cm}^{-1}$ at room temperature downshifts continuously from $1601 \mathrm{~cm}^{-1}$ $\left(-170^{\circ} \mathrm{C}\right)$ to $1594 \mathrm{~cm}^{-1}\left(200^{\circ} \mathrm{C}\right)$, while the Raman band around $1620 \mathrm{~cm}^{-1}$ scarcely changes and (ii) the band due to the terminal PTM radical groups at $1514 \mathrm{~cm}^{-1}$ at room

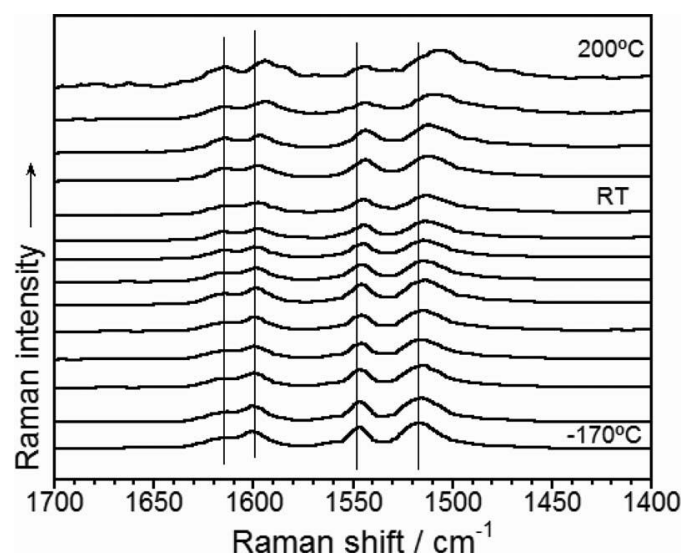

FIG. 10. FT-Raman spectra of $\mathbf{T P M}^{\bullet}-\mathbf{- 1 P V}-\boldsymbol{\bullet}^{\mathbf{T P M}}$ as a function of the temperature. 
temperature also downshifts continuously from $1517 \mathrm{~cm}^{-1}$ $\left(-170{ }^{\circ} \mathrm{C}\right)$ to $1507 \mathrm{~cm}^{-1}\left(200^{\circ} \mathrm{C}\right)$. In general, the thermal cycles are reversible and the room temperature spectra are recovered after the cooling-heating process.

Given the energy proximity between the singlet and triplet diradicals, or small $\Delta\left(\mathrm{S}_{0}-\mathrm{T}_{1}\right)$, one would expect a frequency shift in the spectra with the temperature. On the other hand, from a theoretical point of view the Raman spectra of the singlet and the triplet are almost coincident so that no changes in the intensities are expected with the temperature. This is actually observed in the evolution of the spectra with the temperature which is in accordance with the proximity of the singlet and triplet diradical species. No coincident trends between the thermo-spectroscopic and the high-pressure results are observed, indicating that removing of thermal energy from the materials does not lead to any relevant conformational change.

\section{CONCLUSIONS}

In this paper, a full Raman spectroscopic study is performed on two diradicals formed by PTM radical units connected through $p$-phenylene-vinylene chains of two different lengths and conjugation extensions. Complementary quantum-chemical calculations are reported to interpret the measured Raman spectra. We were also able to distinguish the bands arising from the central bridge and from the external spin bearing moieties. The low-lying electronic absorptions have been described as donor-to-acceptor electronic transitions leading to the use of resonance Raman spectroscopy to extract the vibrational fingerprint involved in these $\pi-\pi^{*}$ electronic excitations. The evolution of the Raman frequencies with the chain length is interpreted by considering the electron-donor character of the bridge and the electronacceptor character of the spin bearing groups. In particular, the changes of the Raman frequencies of the most intense PTM and PPV bands between the two diradicals and of these as a function of the pressure and by heating will be interpreted in terms of the spin delocalization effect. The extension of the $p$-phenylene-vinylene chain produces a downshift of the main Raman band, in accordance with the $\pi$-electron delocalization increment, which also transports the inter-radical spin interaction via a through $\pi$-bond mechanism. This is in good agreement with EPR data on the two studied diradicals that predict inter-radical distances lower than the through-space distances. The conformational effect of the bridge is analyzed by means of pressure studies. Interestingly, pressure induces the planarization of the conjugated linker which, at the same time, favors the inter-radical interaction.

In summary, we show here how $\pi$-electron conjugation within the bridge is instrumental to allow electron and spin communication between the radical centers and how this can be modulated by modifying the bridge conformation (by pressure and heating). In addition, we have extended the suitability of Raman spectroscopy to probe the electronic structure of $\pi$-conjugated molecules $(\pi$-charge delocalization) to the case of $\pi$-spin interactions between unpaired electrons in ground state diradicals via a through-bond channel. In particular, this is possible in the case of spin delocalization mechanism.

\section{ACKNOWLEDGMENTS}

The authors thank EU Large Project ONE-P (FP7-NMP2007-212311), Instituto de Salud Carlos III through "Acciones CIBER," the MICINN, Spain (CTQ2006-06333/BQU and CTQ2010-19501/BQU), and Generalitat de Catalunya (2009SGR516). Financial support by the MICINN of Spain project CTQ2009-10098 and the Junta de Andalucía (Project No. PO9-4708). MICINN is also acknowledged under projects CSD2007-00045 and CTQ2009-14596-C0201/BQU. J.J.N. and F.M. thank support by the Spanish Science and Innovation Ministry, MICINN, (MAT200802032/MAT and UNBA05-33-001), the Catalan Autonomous Government (2009 SGR 1203), and also, the computer time provided by CESCA and BSC.

${ }^{1}$ J. Veciana, J. J. Novoa, M. Deumal, and J. Cirujeda in Magnetic Properties of Organic Materials, edited by P. M. Lathi (Marcel Dekker Inc., New York, 1999).

${ }^{2}$ J. Veciana and H. Iwamura, MRS Bull. 25, 41 (2000).

${ }^{3}$ For a complete series of contributions related with magnetic coupling mechanisms in molecules see: MagnetoScience: Molecules to Materials, edited by J. Miller and M. Drillon (Wiley-VCH, Weinheim, 2001-2006), Vols. 1-6.

${ }^{4}$ V. Polo, A. Alberola, J. Andrés, J. Anthony, and M. Pilkington, Phys. Chem. Chem. Phys. 10, 857 (2008).

${ }^{5}$ M. O. Sandberg, O. Nagao, Z. Wu, M. M. Matsushita, and T. Sugawara, Chem. Commun. 32, 3738 (2008).

${ }^{6}$ P. Wautelet, J. Le Moigne, V. Videka, and P. Turek, J. Org. Chem. 68, 8025 (2003)

${ }^{7}$ R. G. Hicks, Stable Radicals: Fundamentals and Applied Aspects of OddElectron Compounds (John Wiley and Sons, Wiltshire, 2010).

${ }^{8}$ T. Sugawara, H. Komatsu, and K. Suzuki, Chem. Soc. Rev. 40, 3105 (2011).

${ }^{9}$ J. J. Novoa, M. Deumal, and J. Jornet-Somoza, Chem. Soc. Rev. 40, 3182 (2011).

${ }^{10}$ V. Podzorov, E. Menard, A. Borissov, V. Kiryukhin, J. A. Rogers, and M. E. Gershenson, Phys. Rev. Lett. 93, 086602 (2004).

${ }^{11}$ A. Dodabalapur, L. Torsi, and H. E. Katz, Science 268, 270 (1995).

${ }^{12}$ F. Garnier, A. Yassar, R. Hajlaoui, G. Horowitz, F. Deloffre, B. Servet, S. Ries, and P. Alnot, J. Am. Chem. Soc. 115, 8716 (1993).

${ }^{13}$ Organic Spintronics, edited by Z. V. Vardeny (CRC Press, Florida, 2010).

${ }^{14}$ M. Mas-Torrent, N. Crivillers, V. Mugnaini, I. Ratera, C. Rovira, and J. Veciana, J. Mater. Chem. 19, 1691 (2009).

${ }^{15}$ J. H. Burroughes, D. D. C. Bradley, A. R. Brown, R. N. Marks, K. Mackay, R. H. Friend, P. L. Burns, and A. B. Holmes, Nature (London) 347, 539 (1990).

${ }^{16}$ M. M. Wienk and R. A. J. Janssen, J. Am. Chem. Soc. 118, 10626 (1996).

${ }^{17}$ J. R. Sheats, H. Antoniadis, M. Mueschen, W. Leonard, J. Miller, R. Moon, D. Roitman, and A. Stocking, Science 273, 884 (1996).

${ }^{18}$ C. W. Tang, S. A. Van Slyke, and C. H. Chen, J. Appl. Phys. 65, 3610 (1989).

${ }^{19}$ R. F. Service, Science 273, 878 (1996).

${ }^{20}$ J. C. Scott, G. G. Malliaras, W. D. Chen, J.-C. Breach, J. R. Salem, P. J. Brock, S. B. Sachs, and C. E. D. Chidsey, Appl. Phys. Lett. 74, 1510 (1999).

${ }^{21}$ C. Rovira, D. Ruiz-Molina, O. Elsner, J. Vidal-Gancedo, J. Bonvoisin, J. P. Launay, and J. Veciana, Chem. Eur. J. 7, 240 (2001).

${ }^{22}$ O. Elsner, D. Ruiz-Molina, J. Vidal-Gancedo, C. Rovira, and J. Veciana, Nano Lett. 1, 117 (2001).

${ }^{23}$ V. Lloveras, J. Vidal-Gancedo, D. Ruiz-Molina, T. M. Figueira-Duarte, J. F. Nierengarten, J. Veciana, and C. Rovira, Faraday Discuss. 131, 291 (2006).

${ }^{24}$ V. Lloveras, J. Vidal-Gancedo, T. M. Figueira-Duarte, J. F. Nierengarten, J. J. Novoa, F. Mota, N. Ventosa, J. Veciana, and C. Rovira, J. Am. Chem. Soc. 133, 5818 (2011).

${ }^{25}$ G. Zerbi, C. Castiglioni, and M. Del Zoppo, Electronic Materials: The Oligomer Approach (Wiley-VCH, 1998). 
${ }^{26}$ C. Castiglioni, M. Gussoni, J. T. López Navarrete, and G. Zerbi, Solid State Commun. 65, 625 (1988).

${ }^{27}$ J. Kikas, A. Laisaar, A. Suisalu, A. Kuznetsov, and A. Ellervee, Phys. Rev. B 57, 14 (1998).

${ }^{28}$ S. C. Yang, W. Graupner, S. Guha, P. Puschnig, C. Martin, H. R. Chandrasekhar, M. Chandrasekhar, G. Leising, C. Ambrosch-Draxl, and U. Scherf, Phys. Rev. Lett. 85, 2388 (2000).

${ }^{29}$ S. Guha, W. Graupner, R. Resel, M. Chandrasekhar, H. R. Chandrasekhar, R. Glaser, and G. Leising, Phys. Rev. Lett. 82, 3625 (1999).

${ }^{30}$ B. Toudic, P. Limelette, G. Froyer, F. Le Gac, A. Moréac, and P. Rabileller, Phys. Rev. Lett. 95, 215502 (2005).

${ }^{31}$ J. Schmidtke, J. S. Kim, J. Gierschner, C. Silva, and R. H. Friend, Phys. Rev. Lett. 99, 167401 (2007).

${ }^{32}$ R. Ponce Ortiz, J. Casado, V. Hernández, J. T. López Navarrete, P. M. Viruela, E. Ortí, K. Takimiya, and T. Otsubo, Angew. Chem. Int. Ed. 46, 9057 (2007).

${ }^{33}$ J. Casado, S. Patchkovskii, M. Z. Zgierski, L. Hermosilla, C. Sieiro, M. Moreno Oliva, and J. T. López Navarrete, Angew. Chem. Int. Ed. 47, 1443 (2008).

${ }^{34}$ V. G. Baonza, M. Taravillo, A. Arencibia, M. Cáceres, and J. Núñez, J. Raman Spectrosc. 34, 264 (2003).

${ }^{35}$ G. J. Piermarini, S. Block, J. D. Barnett, and J. A. Forman, J. Appl. Phys. 46, 2774 (1975).

${ }^{36}$ J. K. Labanowski and J. W. Andzelm, Density Functional Methods in Chemistry (Springer, New York, 1991).

${ }^{37}$ M. J. Frisch, G. W. Trucks, H. B. Schlegel et al., GAUSSIAN03, Revision C.02 Gaussian Inc., Walingford, CT, 2003.
${ }^{38}$ A. D. Becke, J. Chem. Phys. 98, 5648 (1993).

${ }^{39}$ C. Lee, W. Yang, and R. G. Parr, Phys. Rev. B 37, 785 (1988).

${ }^{40}$ J. H. Los and A. Fasolino, Phys. Rev. B 68, 24107 (2003).

${ }^{41}$ M. M. Francl, W. J. Pietro, W. J. Hehre, J. S. Binkley, M. S. Gordon, D. J. Defrees, and J. A. Pople, J. Chem. Phys. 77, 3654 (1982).

${ }^{42}$ Some examples of the application of the broken-symmetry approach to diradicals: A. Ehesan and S. Datta, J. Phys. Chem. A 110, 2776 (2006); M. Mitani, H. Mori, Y. Takano, D. Yamaki, Y. Yoshioka, and K. Yamaguchi, J. Chem. Phys. 113, 4035 (2000).

${ }^{43}$ A. P. Scott and L. Radom, J. Phys. Chem. 100, 16502 (1996).

${ }^{44} \mathrm{~K}$. Müllen and G. Wegner, Electronic Materials: The Oligomer Approach (Wiley-VCH, Weinheim, 1998).

${ }^{45}$ I. Ratera, D. Ruiz-Molina, F. Renz, J. Ensling, K. Wurst, C. Rovira, and J. Veciana, J. Am. Chem. Soc. 125, 1462 (2003); C. Sporer, I. Ratera, D. Ruiz-Molina, Y. Zhao, J. Vidal-Gancedo, K. Wurst, P. Jaitner, K. Clays, A. Persoons, C. Rovira, and J. Veciana, Angew. Chem. Int. Ed. 43, 5266 (2004); I. Ratera, C. Sporer, D. Ruiz-Molina, N. Ventosa, J. Baggerman, A. Brouwer, C. Rovira, and J. Veciana, J. Am. Chem. Soc. 129, 6117 (2007); G. D'Avino, L. Grisanti, J. Guasch, I. Ratera, J. Veciana, and A. Painelli, ibid. 130, 12064 (2008).

${ }^{46}$ S. D. McGlynn, T. Azumi, and M. Kinoshita, Molecular Spectroscopy of the Triplet State (Prentice Hall, New Jersey, 1969).

${ }^{47}$ P. Wautelet, L. Catala, A. Bieber, P. Turek, and J. J. André, Polyhedron 20, 1571 (2001)

${ }^{48}$ S. Webster and D. N. Batchelder, Polymer 37, 4961 (1996).

${ }^{49}$ See supplementary material at http://dx.doi.org/10.1063/1.4871895 for theoretical Raman spectra with different level of calculations and vibrational eigenvectors. 